\title{
A multicenter study of endocrine abnormalities in septo-optic dysplasia (SOD) in Asean countries
}

\author{
Suttipong Wacharasindhu ${ }^{1 *}$, Pattareeya Yottasan ${ }^{1}$, Somchit Jaruratanasirikul ${ }^{1}$, Ouyporn Panamonta', \\ Kevalee Unachak', Aman Pulungan², Muhammad Faizi ${ }^{2}$, Nur Rochmah², Susana Campos ${ }^{3}$, SiokSoan Chan Cua ${ }^{3}$, \\ Fatimah Binti Harun ${ }^{4}$, Muhammad Yazid Jalaludin ${ }^{4}$, Vu Chi Dung Noi tiet ${ }^{5}$
}

From 8th APPES Biennial Scientific Meeting

Darwin, Australia. 29 October - 1 November 2014

\section{Background}

Septo-optic dysplasia (SOD) is a heterogeneous malformation condition consisting of optic nerve hypoplasia, various types of forebrain defects and hormonal deficiencies. This study aims to expand knowledge about endocrine abnormalities in patients with SOD in ASEAN countries.

\section{Material and method}

Forty-eight patients (27 male, 21 female) who has been diagnosed as having SOD in ASEAN countries were clinically reviewed from medical records.

\section{Results}

Clinical manifestations and endocrine abnormalities of the patients are shown in Table 1 .

\section{Conclusion}

This multicenter and multinational study shows that about 20-35 \% of SOD patients have endocrine abnormalities. Hypothyroidism and GH insufficiency are the most common endocrine problems associated with this condition.

\section{Table 1 Clinical manifestation of SOD patients}

\begin{tabular}{|c|c|c|c|c|c|}
\hline \multicolumn{3}{|c|}{ Clinical manifestation } & \multicolumn{3}{|l|}{ Clinical manifestation } \\
\hline \multicolumn{2}{|c|}{$\begin{array}{l}\text { Age at presentation (months) mean } \pm \\
\text { SD }\end{array}$} & $33 \pm 39$ months (range $20-178$ ) & MRI findings & Absence of septum pellucidum(\%) & 62 \\
\hline \multicolumn{2}{|c|}{ Ht SDS, mean \pm SD } & $-0.97 \pm 1.97$ & & Pituitary hypoplasia (\%) & 33 \\
\hline \multicolumn{2}{|c|}{ Wt SDS, mean \pm SD } & $0.02 \pm 2.35$ & & Abnormal of corpus callosum (\%) & 16 \\
\hline \multirow[t]{3}{*}{ Eye presentation } & Nystagmus (\%) & 54 & & Cortical dysplasia (\%) & 8 \\
\hline & Squint (\%) & 3 & Endocrine abnormalities & Hypothyroid (\%) & 35 \\
\hline & Poor vision (\%) & 35 & & GH insufficiency (\%) & 29 \\
\hline \multicolumn{2}{|c|}{ Delayed development (\%) } & 16.2 & & Delayed/arrested puberty/suspected HG (\%) & 23 \\
\hline \multicolumn{2}{|l|}{ Seizure (\%) } & 7.9 & & Cortical insufficiency (\%) & 17 \\
\hline \multicolumn{2}{|l|}{ Hypoglycemia (\%) } & 8.1 & & Diabetes insipidus (\%) & 19 \\
\hline \multicolumn{2}{|c|}{ Neonatal jaundice (\%) } & 2.7 & Eye examination & Unilat ON hypoplasia & 13 \\
\hline \multicolumn{2}{|c|}{ Undescended testes (\%) (boys) } & 5.4 & & Bilat ON hypoplasia & 84 \\
\hline \multicolumn{2}{|c|}{ Micropenis (\%) (boys) } & 21 & & Microcornea & 3 \\
\hline
\end{tabular}

HG = Hypogonadism, ON = Optic nerve

${ }^{1}$ A ASEAN Study group, Thailand

Full list of author information is available at the end of the article

(c) 2015 Wacharasindhu et al; licensee BioMed Central Ltd. This is an Open Access article distributed under the terms of the Creative 


\section{Authors' details}

${ }^{1}$ A ASEAN Study group, Thailand. ${ }^{2}$ A ASEAN Study group, Indonesia.

${ }^{3}$ A ASEAN Study group, Philippines. ${ }^{4}$ A ASEAN Study group, Malaysia.

${ }^{5}$ A ASEAN Study group, Vietnam.

Published: 28 April 2015

doi:10.1186/1687-9856-2015-S1-P85

Cite this article as: Wacharasindhu et al:: A multicenter study of

endocrine abnormalities in septo-optic dysplasia (SOD) in Asean countries. International Journal of Pediatric Endocrinology 20152015

(Suppl 1):P85.

Submit your next manuscript to BioMed Central and take full advantage of:

- Convenient online submission

- Thorough peer review

- No space constraints or color figure charges

- Immediate publication on acceptance

- Inclusion in PubMed, CAS, Scopus and Google Scholar

- Research which is freely available for redistribution

Submit your manuscript at 